\title{
Considerations regarding a diagnosis of Alzheimer's disease before dementia: a systematic review
}

\author{
Jetske van der Schaar ${ }^{*}$ (i), Leonie N. C. Visser ${ }^{1,2}$, Femke H. Bouwman', Johannes C. F. Ket ${ }^{3}$, Philip Scheltens ${ }^{1}$,
} Annelien L. Bredenoord ${ }^{4}$ and Wiesje M. van der Flier ${ }^{1,5}$

\begin{abstract}
Background: The NIA-AA research framework proposes a purely biological definition of Alzheimer's disease (AD). This implies that AD can be diagnosed based on biomarker abnormalities, irrespective of clinical manifestation. While this brings opportunities, it also raises challenges. We aimed to provide an overview of considerations regarding the disclosure of AD pathology before the onset of dementia.

Methods: A systematic literature review was conducted and reported according to PRISMA guidelines. We searched PubMed, Embase, APA PsycINFO, and Web of Science Core Collection (on 10 December 2020) for references on conveying AD biomarker results to individuals without dementia. Our query combined variations on the terms Alzheimer's disease, disclosure, or diagnosis, preclinical or prodromal, and biomarkers. Two reviewers independently screened the resulting 6860 titles and abstracts for eligibility and examined 162 full-text records for relevance. We included theoretical articles in English, on communicating amyloid and/or tau results to individuals with mild cognitive impairment, subjective cognitive decline, or normal cognition. MAXQDA-software was used for inductive data analysis.
\end{abstract}

Results: We included 27 publications. From these, we extracted 26 unique considerations, which we grouped according to their primary relevance to a clinical, personal, or societal context. Clinical considerations included (lack of) validity, utility, and disclosure protocols. Personal considerations covered psychological and behavioral implications, as well as the right to (not) know. Finally, societal considerations comprised the risk of misconception, stigmatization, and discrimination. Overall, views were heterogeneous and often contradictory, with emphasis on harmful effects.

Conclusions: We found 26 diverse and opposing considerations, related to a clinical, personal, or societal context, which are relevant to diagnosing AD before dementia. The theoretical literature tended to focus on adverse impact and rely on common morality, while the motivation for and implications of biomarker testing are deeply personal. Our findings provide a starting point for clinicians to discuss biomarker-based diagnosis with their patients, which will become even more relevant in light of the conditional approval of a first disease-modifying drug for AD.

Keywords: Alzheimer's disease, Biomarkers, Diagnosis, Disclosure, Preclinical, Prodromal, Predementia, Ethics

\footnotetext{
*Correspondence: jetske.vanderschaar@amsterdamumc.nl ${ }^{1}$ Alzheimer Center Amsterdam, Department of Neurology, Amsterdam Neuroscience, Vrije Universiteit Amsterdam, Amsterdam UMC, De Boelelaan 1118, 1081, HZ, Amsterdam, The Netherlands

Full list of author information is available at the end of the article
}

\section{Introduction}

The pathophysiological cascade of events in Alzheimer's disease $(\mathrm{AD})$ starts 20 to 30 years before dementia [1-3]. Nowadays, it is possible to detect this pathology in vivo, original author(s) and the source, provide a link to the Creative Commons licence, and indicate if changes were made. The images or other third party material in this article are included in the article's Creative Commons licence, unless indicated otherwise in a credit line to the material. If material is not included in the article's Creative Commons licence and your intended use is not permitted by statutory regulation or exceeds the permitted use, you will need to obtain permission directly from the copyright holder. To view a copy of this licence, visit http://creativecommons.org/licenses/by/4.0/. The Creative Commons Public Domain Dedication waiver (http://creativeco mmons.org/publicdomain/zero/1.0/) applies to the data made available in this article, unless otherwise stated in a credit line to the data. 
using biomarkers. The National Institute on Aging and Alzheimer's Association research framework operationalized $\mathrm{AD}$ as a biological construct characterized by evidence of amyloid plaques, tau tangles, and neurodegeneration, irrespective of clinical expression [4]. This implies that $\mathrm{AD}$ can be diagnosed before dementia, in individuals with mild cognitive impairment, subjective cognitive decline, or normal cognition.

This development has sparked a heated debate. Should a specialist in a memory clinic tell individuals without dementia that they have AD? This may lead to distress [5] as a precise prognosis cannot be given and there is no curative therapy (yet). Alternatively, can physicians withhold information on an underlying disease from patients, just because they do not fulfill clinical dementia criteria? While perhaps avoiding anxiety, this would deprive patients of the opportunity to adopt a risk-reducing lifestyle, prepare for the future, or participate in dementiaprevention trials [6].

The numbers of persons living with preclinical AD are large [7] and many wish to learn their biomarker results [8]. A first estimation suggests the prevalence of AD may be three times higher when based on a biological rather than a clinical definition of the disease, illustrating the potential magnitude of consequences [9]. With the conditional approval of a first disease-modifying therapy [10], this discussion is more relevant than ever.

We aim to provide an overview of ethical, psychosocial, and societal considerations regarding the disclosure of AD pathology before dementia.

\section{Methods}

A systematic literature search was conducted and reported according to PRISMA guidelines [11]. Our broad query combined synonyms and spelling variations on the terms "Alzheimer's" AND "disclos" OR "diagnos" AND "predementia" AND "biomarkers," using controlled standardized keywords as well as free text terms. We searched PubMed, Embase, APA PsycINFO, and Web of Science Core Collection for references published before 10 December 2020 and scanned reference lists of identified articles.

All articles in English presenting theoretical data, i.e., ethical concerns, psychosocial consequences, and societal implications of disclosing amyloid and/or tau results to individuals in $\mathrm{AT}(\mathrm{N})$ stages 1-3 [4] were eligible (provided the full text was available), except (sections of) books, editorials, commentaries, and conference proceedings. Publications on later stages and other types of dementia or neurodegenerative diseases were excluded, as well as those primarily focused on trial design or genetic risk.
Two authors independently screened all titles and abstracts. Articles marked as potentially relevant were assessed for eligibility based on full text. In case of discrepancy, arguments for inclusion and exclusion were discussed while re-examining the contents and criteria. In each case, consensus was reached, without having to consult a third author.

We performed inductive content analysis [12], using MAXQDA-software. First, all sections addressing ethical, psychosocial, and societal considerations regarding the disclosure of $\mathrm{AD}$ pathology before dementia were identified. Next, the data were processed in an iterative and incremental manner to highlight all aspects pertinent. These were further grouped and aggregated in a hierarchy of categories. This process was repeated and revised until the contents were precisely described and fully covered, by a structure of considerations, with underlying arguments and overarching contexts. An additional classification was made according to the key ethical principles of medical ethics: beneficence, non-maleficence, justice, and autonomy [13].

\section{Results}

Our initial search yielded 6860 records. Two reviewers independently screened all titles and abstracts for eligibility and examined 162 full-text records for relevance. After applying selection criteria (Fig. 1), we included 27 articles.

From these, 26 unique considerations were extracted (Table 1), along with supporting arguments, and further categorized according to their primary relevance to a clinical, personal, or societal context. The final coding tree corresponds to the structure of Table 1.

\section{Clinical considerations (Lack of) clinical validity}

Although most authors acknowledged that biomarker information enhances diagnostic accuracy [14-25] and validity is strong in selected cohorts [17-21, 26, 27], the predictive value was debated [14-36]. About half of the articles covered the difficulty to discern normal aging from latent $\mathrm{AD}[14-16,18,20,22,26,27,29-31,33$, $34,37]$, as the majority of cognitively healthy older persons with abnormal biomarkers never develop dementia $[14-16,20-23,26,27,29-31,33,34,38]$, since AD is multi-factorial $[16,18,26,27,31,35]$. It was argued that procedures are not without burden or risk $[14,16,18,20$, $21,23,24,34]$ and consequences of incorrectly labeling people as "patients in waiting" [34] could be severe for the individuals and relatives concerned [14, 16, 24-28, 31, 36]. Most authors concluded that biomarker criteria require final demonstration of validity in populations without dementia [14-23, 26, 27, 30, 31, 33-36, 39]. 


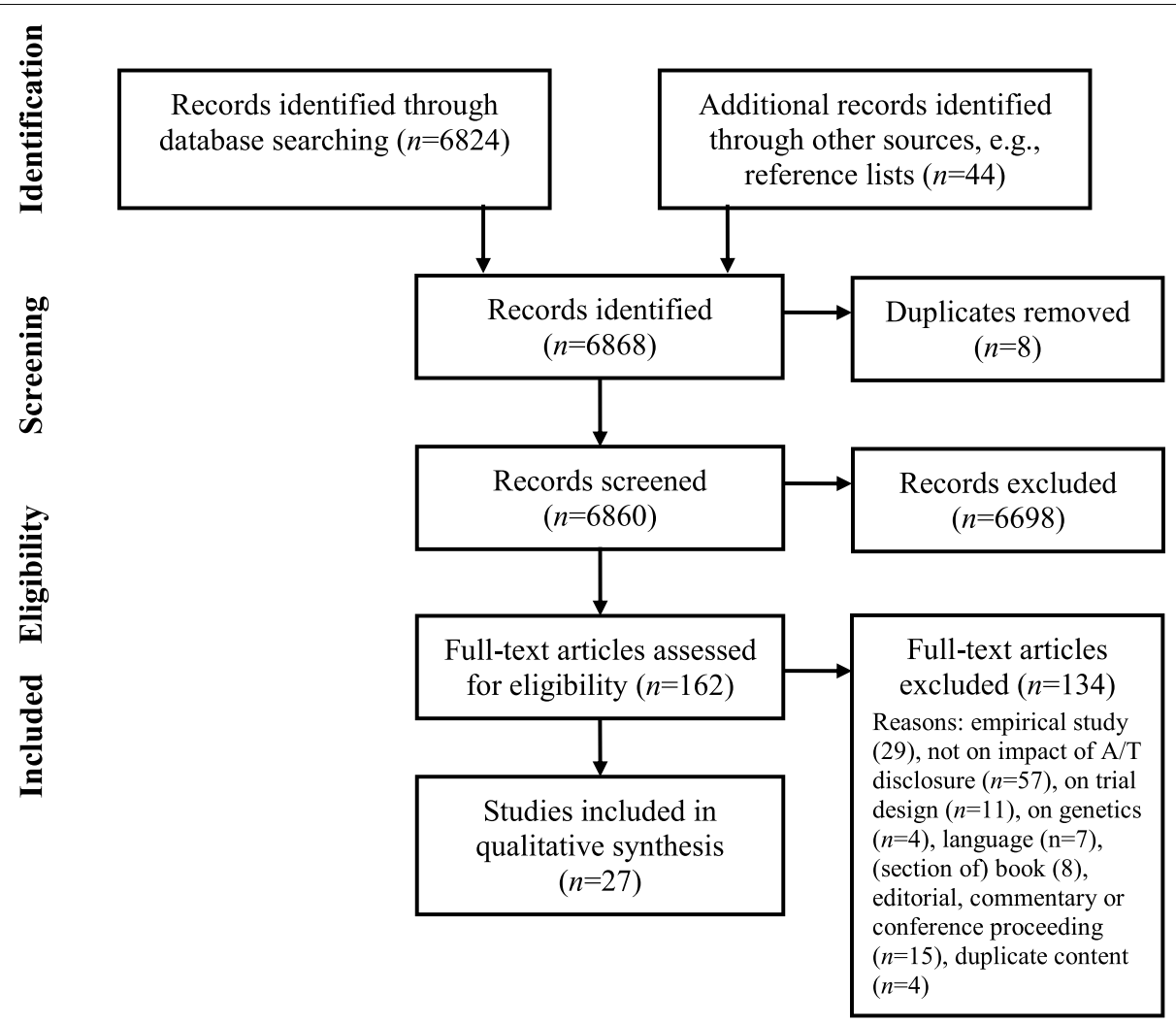

Fig. 1 Flow diagram of study selection

\section{(Lack of) clinical utility}

Clinical utility was contested, mainly due to the absence of a disease-modifying therapy $[14-16,18,20,21,23-$ $25,27,28,31,34-36,38,40]$, and limited effectiveness of symptom suppressing medications, which has not been demonstrated in predementia stages of $\mathrm{AD}[21,24$, 40]. However, several authors suggested lifestyle interventions could delay cognitive impairment $[14,16,17$, 21-32, 34, 36], although evidence on their effectiveness remains inconclusive [14, 15, 21, 31, 36], and according to some, such health improvements should be pursued regardless of one's biomarker status [16, 23, 31]. Lastly, it was argued that early detection can improve patient care, e.g., by offering an explanation for concerns, anticipating medical needs, and facilitating access to support [14, 16, $18,19,22-25,27,28,32,35]$. Nonetheless, others did not consider biomarker information medically meaningful $[15,16,20-26,29-32,36,40]$.

\section{Protocols and training}

A dozen papers addressed the need for guidance regarding who to test, what findings signify, whether to disclose, and how [17, 18, 20-22, 25, 28, 31, 36-39]. Knowledge from the fields of oncology and genetic testing may offer a good starting point $[14,20,23,25,27,28,30,36-40]$.
The literature referred to the development of standardized processes and materials for disclosing biomarker levels in prevention trials $[17,30,32,37,38]$, but reported "a complete lack of studies in a clinical setting" [17]. In addition, it was emphasized that the required skills cannot be assumed, so medical professionals would profit from training in risk communication $[14,16,18,20,22$, $25,28,37,38]$.

\section{Disclosure}

Conveying an $\mathrm{AD}$ diagnosis was considered daunting, especially to persons with full insight $[15,17,20-24,29$, $30,40]$, as knowledge of the impact is scarce, yet urgently required [14-17, 20, 22-25, 27, 30-32, 34, 37, 38]. Recommendations derived from research on genetic risk covered informed consent, pre-test screening, education and counseling, sufficient time for consultation and reflection, take-home materials, involving a relative, and tailoring to individuals $[14,15,17,19-22,24,25,27,28$, 30-32, 35-39].

\section{Personal considerations (Un)certainty}

While an abnormal biomarker result provides certainty on the presence of brain lesions, it was also argued to 
Table 1 Clinical, personal, and societal considerations

\begin{tabular}{|c|c|c|}
\hline & Consideration & Refs \\
\hline & Clinical & \\
\hline 1 & Validity & [14-28] \\
\hline 2 & Lack of validity & [14-39] \\
\hline 3 & Utility & {$[14,16-19,21-24,26-32,34-36,40]$} \\
\hline 4 & Lack of utility & {$[14-18,20-24,26-32,34-36,38,40]$} \\
\hline
\end{tabular}

$5 \quad$ Protocols and training

6 Disclosure

Personal

7 Certainty

8 Uncertainty

$9 \quad$ Actionability (personal utility)

$[14-25,27,28,31,32,34-39]$

10 Lack of actionability (personal utility) $\quad[15,16,23,25,28,32,34]$

11 Positive psychological impact

$[14-20,22-24,27,32,36]$

\section{Arguments}

- Clinical criteria have limited validity

- Biomarkers enhance diagnostic certainty and accuracy

- Validity is strong or sufficient (in selected cohorts)

- Predictive value for progression is demonstrated (in selected cohorts)

- Validity is uncertain and insufficient or needs further research

- Predictive value is uncertain and limited or needs further research

- Discerning normal aging from latent disease is difficult or impossible

- Many individuals with AD biomarkers never develop dementia

- Abnormal biomarkers are not the sole cause of AD

- Procedures may be burdensome or risky

- Consequences of misdiagnosis are severe

- Drugs can suppress symptoms in some patients

- Lifestyle changes or interventions may be beneficial

- Early diagnosis may lead to better healthcare

- Utility is absent and uncertain or needs further research

- There is no disease-modifying therapy

- Lifestyle changes or interventions have uncertain or modest effect at best

- Lifestyle changes should be done regardless

- Protocols, methods, and materials are scarce or required - Knowledge of genetic or oncological markers may offer a starting point

- Healthcare professionals need to develop knowledge and skills

- There is growing consensus toward the use of biomarkers and sharing of results

- Disclosure is difficult, especially to a person with (full) insight

- Safety may be improved by informed consent, pre-test counseling, post-disclosure support, take-home materials, time for reflection, involvement of a relative, and tailoring the approach to the individual's needs

- Research into the impact of disclosure is scarce or required

- An in vivo (biomarker) diagnosis of $A D$ is inherently uncertain

- Individuals may appreciate an uncertain risk prediction - Individuals may understand uncertainty

- Early detection may lead to more or longer uncertainty - Individuals may expect a certain diagnosis

- Individuals may misinterpret test results

- It is hard to deal and live with uncertainty

- Individuals and relatives can prepare for the future

- Individuals can advance plans or improve quality of life

- Individuals can contribute to or profit from prevention trials

- There is no actionability

- Results may be too uncertain for decisions

- Preparing for the future should be done regardless

- Result may lead to positive emotions, e.g., relief, solace, or social exoneration (by providing an explanation for behavior or functioning)

- There is value in knowing, understanding, and accepting the situation 
Table 1 (continued)

\begin{tabular}{|c|c|c|c|}
\hline & Consideration & Refs & Arguments \\
\hline 12 & Negative psychological impact & {$[14,16-18,20-32,34-40]$} & $\begin{array}{l}\text { - Result may lead to negative emotions, e.g., fear, anxiety, } \\
\text { and depression } \\
\text { - There is risk of catastrophic reactions (euthanasia or } \\
\text { suicide) } \\
\text { - Negative reactions may be over-rated, limited, or prevent- } \\
\text { able } \\
\text { - Early detection may increase (subjective) cognitive } \\
\text { decline (stereotype threat) }\end{array}$ \\
\hline 13 & Right to (not) know & {$[14,15,17,19-25,27-35,39]$} & $\begin{array}{l}\text { - Individuals have a right to know their status in research } \\
\text { and the clinic } \\
\text { - Individuals have a right to not know their status in } \\
\text { research and the clinic } \\
\text { - Right to (not) know may be overruled by the principle of } \\
\text { primum non nocere (first, do no harm) }\end{array}$ \\
\hline \multirow[t]{2}{*}{14} & Wish to (not) know & {$[14-22,24,25,28,29,31,32,35,36,38,39]$} & $\begin{array}{l}\text { - Many individuals wish to know } \\
\text { - Some individuals do not wish to know } \\
\text { - Individual's preference should be included in the } \\
\text { informed consent }\end{array}$ \\
\hline & Societal & & \\
\hline 15 & Share & {$[14,16,19-21,23,24,28,30-33,35,37-39]$} & $\begin{array}{l}\text { - Patients have the right to privacy and confidentiality } \\
\text { - Fear of stigma and discrimination may prevent individuals } \\
\text { from seeking help } \\
\text { - A predementia diagnosis may lead to support }\end{array}$ \\
\hline 16 & Support & {$[14,20,22,28,30,32,37]$} & $\begin{array}{l}\text { - Services are needed to help individuals cope with prede- } \\
\text { mentia AD }\end{array}$ \\
\hline 17 & Stigma & {$[14,16,20-26,28-30,32,34,35,37,38,40]$} & $\begin{array}{l}\text { - Label of AD may lead to loss of status, identity, or person- } \\
\text { hood } \\
\text { - Label of AD may lead to public stigma, e.g., pity, patron- } \\
\text { izing, and distancing } \\
\text { - Label of AD may lead to self-stigma } \\
\text { - Label of AD may lead to spillover stigma (extends to } \\
\text { relatives) } \\
\text { - Predementia detection may increase the stigma of AD } \\
\text { - Predementia detection may decrease the stigma of AD }\end{array}$ \\
\hline 18 & Discrimination & {$[14,16,17,20-24,27-33,35,37-40]$} & $\begin{array}{l}\text { - Predementia detection may lead to discrimination in } \\
\text { employment, insurance, rights (voting and driving), health } \\
\text { care, legal status }\end{array}$ \\
\hline 19 & Policy and law & {$[14,20-25,28,30-33,35,37-39]$} & $\begin{array}{l}\text { - Current laws do not protect individuals from biomarker } \\
\text { discrimination } \\
\text { - Regulation and law should regulate confidentiality and } \\
\text { privacy } \\
\text { - Regulation and law should regulate obligation to (not) } \\
\text { disclose } \\
\text { - Regulation and law should regulate forced screening or } \\
\text { testing }\end{array}$ \\
\hline 20 & Misconception & {$[14-17,19-25,27-32,34-38]$} & $\begin{array}{l}\text { - The concept of predementia AD is difficult to explain and } \\
\text { understand } \\
\text { - (Re) conceptualization of AD may lead to misconceptions } \\
\text { - At-risk individuals are prone to (therapeutic) misconcep- } \\
\text { tions } \\
\text { - Healthcare professionals are prone to misinterpretation } \\
\text { - The general public is prone to misconceptions }\end{array}$ \\
\hline 21 & Engagement and education & {$[14,16,20-22,24-26,28,30-32,35,37-39]$} & $\begin{array}{l}\text { - Patients and the public should be involved in the design } \\
\text { of protocols and policy } \\
\text { - Education may improve awareness, acceptance, and } \\
\text { attitudes }\end{array}$ \\
\hline 22 & Advance research & {$[14-16,18,20,22,23,27-35,37,38,40]$} & $\begin{array}{l}\text {-Therapies are more likely to prevent than cure AD } \\
\text { - Predementia phase offer opportunities to stop, delay, or } \\
\text { slow symptom onset } \\
\text { - Potential interventions will target patients in early stages } \\
\text { of the disease }\end{array}$ \\
\hline
\end{tabular}


Table 1 (continued)

\begin{tabular}{|c|c|c|c|}
\hline & Consideration & Refs & Arguments \\
\hline 23 & Medicalization & {$[14,20,23,28,32,34,35,37]$} & $\begin{array}{l}\text { - Predementia detection may lead to medicalization and } \\
\text { overdiagnosis } \\
\text { - Predementia detection may increase urgency or treating } \\
A D \\
\text { - Predementia detection may decrease urgency or treating } \\
\text { AD }\end{array}$ \\
\hline 24 & Resources & {$[14,16-18,20,22-24,27,28,30-33,35,37,39]$} & $\begin{array}{l}\text { - Tests and treatment may not be affordable or accessible } \\
\text { for all } \\
\text { - Predementia detection may exhaust healthcare and } \\
\text { overwhelm current systems } \\
\text { - Predementia detection may prioritize prevention over } \\
\text { care or other research } \\
\text { - Early detection may lead to higher cost } \\
\text { - Early detection may lead to lower cost }\end{array}$ \\
\hline 25 & Hope & {$[23,28,34]$} & $\begin{array}{l}\text { - Research (participation) should not be motivated by false } \\
\text { hope }\end{array}$ \\
\hline 26 & Fear & {$[14,16,23,24,26,29,31,32,34,35,37,39]$} & $\begin{array}{l}\text { - There is a lot of fear for (the implications) of AD } \\
\text { - Predementia detection may increase fear }\end{array}$ \\
\hline
\end{tabular}

Considerations according to context. By inductively analyzing the literature presenting theoretical data on disclosing the presence of AD pathology to individuals without dementia, we extracted 26 unique considerations from 25 articles. We categorized these considerations according to the context they primarily related to, i.e., clinical, personal, and societal context, and collected the underlying arguments as stated by the authors

cause uncertainty about the eventuality of cognitive decline $[14,20,23-25,32,34]$, which was suggested to be hard to deal with $[20,21,32]$. While a few authors felt individuals might appreciate and understand such indefinite risk [17, 23, 34, 37, 39], the majority feared that it would be misinterpreted as an inevitability $[14,15,20,22$, $24,28,31,35,36]$

\section{(In)actionability}

Proponents stated that awareness of having AD without dementia allowed individuals and relatives to prepare for the future by arranging private, professional, financial, and legal matters; obtaining long-term care insurance; writing advance directives or making end-of-life decisions [14-25, 27, 28, 31, 32, 34, 36-39]; participating in prevention trials [14-18, 20, 22-24, 35, 38]; or retiring early and enjoying time left $[14,23]$. Opponents believed these things should be done anyway $[15,16,23]$ and questioned whether the predictive power was sufficient to substantiate far-reaching decisions [15, 25, 28, 32].

\section{Positive and negative psychological impact}

Several publications observed the absence and even presence of AD lesions might offer relief, solace, or an explanation of symptoms [17-19, 22-24, 27, 32, 36]. Knowing what is going on was thought to be of value in itself [14-17, 20, 22-24, 27, 32]. Yet, nearly all papers mentioned adverse emotions, including fear, anxiety, and depression $[14,16-18,20-32,34-39]$, or catastrophic reactions, e.g., suicide $[17,18,20,22,23,25$, $28,30,32,35,36,38-40]$, despite reason to believe negative reactions may be over-rated, limited, or temporary [17, 18, 20, 22, 23, 25, 28, 30, 32, 35, 36, 38, 39]. Another worry was the risk of stereotype threat or nocebo reaction, where the knowledge of susceptibility leads to the associated behavior or a decrease in memory functioning $[14,17,23,28,31,34,37]$.

\section{Right to (not) know}

On moral and legal grounds, an individual's request to access one's personal data must be granted, be it in a research trial or clinical practice $[15,17,19-25,27-29$, $31,34,39]$. Likewise, a refusal to be informed of such information must be respected as well [14, 15, 20, 21, 23-25, 28, 35]. However, it was argued that this fundamental right can be in conflict with physicians' oath of primum non nocere (first, do no harm) and in some cases could or even should be overruled [14, 17, 19-24, 27-33].

\section{Wish to (not) know}

Authors reported that many individuals express a desire to receive risk information $[15-17,22,24,25,28$, $29,31,32,36,39]$, while some might prefer to remain ignorant of uncertain odds $[16,17,21,24,29,32]$. It was recommended that extensive and "truly" informed consent [31] should record a patient's preference and list which other persons and authorities will be notified $[14,15,18,20,21,24,28,31,32,35,38,39]$. 


\section{Societal considerations \\ Sharing}

As individuals have the right to privacy and confidentiality [14, 19-21, 23, 24, 28, 30-33, 35, 37-39], it was emphasized that diagnostic information should not be released to relatives [24, 35] or third parties [14, 20, $21,23,24,30-33,35,37,39]$ without their consent and against their interest, although in case of driving, physicians may be obliged to report this to relevant authorities [38]. It was also mentioned that fear of stigma could prevent patients from voicing their concerns and seeking help, while acting on their worries and needs may also yield support [14, 16, 20, 23, 28, 32, 35, 37].

\section{Support services}

Apart from pre- and post-diagnostic counseling [14, 17, 20-22, 24, 28, 30-32, 35, 37-39], literature addressed the need of support services for people along the continuum of $\mathrm{AD}$, including assistance in personal, social, and healthcare needs and monitoring of professional, financial, and legal capacities $[14,20,22,26,28,30,32$, 37, 39].

\section{Stigma}

According to the identified literature, public stigma ranges from patronizing attitudes to social distancing, exclusion, and isolation $[14,23,25,26,28-30,32,34$, $37,38,40]$. This induces self-stigma, when pejorative views are internalized as feelings of shame, lowered selfesteem, and inferiority [14, 23, 30, 32, 34, 37]. In addition, spillover stigma is detrimental to family members [14, $28,29,32,37]$. An increase of predementia patients was expected to expand stigma [14, 23, 30, 34, 37, 38]; conversely, normalization was assumed to dilute it as well $[14,20,37]$.

\section{Discrimination}

Individuals with an $\mathrm{AD}$ diagnosis and risk of dementia were considered vulnerable to discrimination, affecting their professional position, insurance fees, legal status, civil rights (driving and voting), and financial capacity $[14,16,17,20-24,27-33,35,37-40]$.

\section{Policy and law}

Current legislation, such as the United States Genetic Information Non-Discrimination Act and the Americans with Disabilities Act, does not adequately protect individuals with predementia $\operatorname{AD}[20,22,28,33,37,38]$. Several authors advocated for regulation of confidentiality and privacy, preclinical screening, and obligatory disclosure for persons with high responsibility [14, 20, 21, 23-25, 28, 30-33, 35, 37-39].

\section{Misconception}

Authors observed that a symptomless condition with an uncertain prognosis is hard to grasp for lay persons and medical professionals alike $[14,17,20,22-24,28,29$, $35,36]$, and the changing meaning of "AD" could lead to incorrect interpretations [14, 21, 24, 29, 30, 32, 34], especially since at-risk individuals are prone to misconceptions [14-17, 22, 23, 28, 31, 34-36], healthcare providers apply different interpretations of disease criteria $[14,16,17,19,22,27-29,31]$, and the general public is influenced by dementia myths and the media's portrayal of AD patients as "dehumanized shells" [14, 25, 30, 37].

\section{Education and engagement}

Several publications stress that individuals' perceptions can change after intervention or experience $[15,17,28$, $31,34,38]$, and public dialogue may improve awareness and attitudes [14, 22, 24-26, 28, 30, 31, 35, 37, 39]. Moreover, patients of all cultures should be involved in the development of protocols and policy, to represent their own views, improve research, and decrease stigma [14, $16,20-22,24,25,28,30,32,35,37,40]$.

\section{Resources, opportunities, and costs}

Authors worried that predementia testing may not be accessible and affordable for all [14, 16, 23, 24, 27, 30, 37], individuals with minimal symptoms could strain healthcare services $[14,22,23,37]$, and a focus on prevention research might come at the expense of patients with advanced $\operatorname{AD}[14,16,20,35,37]$. Thus, the emotional and financial burden could rise substantially [14, 16-18, 20, $23,24,30-33,35,37$ ] or drop considerably when patients live longer at home, at-risk participants lower trial costs, and medication becomes available $[14,16,18,20,23,27$, 28, 32, 39].

\section{Medicalization}

Expanding the criteria for AD raised concerns of tipping the scales from under- to overdiagnosis $[14,20,23,28$, $32,35,37]$. Paradoxically, normalization was reasoned to result in marginalization, but also argued to increase the urgency to develop disease-modifying therapies [14].

\section{Advance research}

The primary consideration behind the new criteria is to prevent individuals with AD from developing dementia, as early interventions are hypothesized to have better chances of success [14-16, 18, 20, 22, 23, 27-35, 37, 38, 40]. 


\section{Hope and fear}

Finally, authors were wary of inflating unsubstantiated hope [23, 28, 34] and/or further fueling already widespread fear [14, 16, 23, 24, 26, 29, 31, 32, 34, 35, 37, 39]. Both were primarily regarded as vulnerabilities and impediments to rational decision-making [23, 34, 35].

\section{Key principles}

In substantiating their arguments, the majority of authors invoked the key principles of medical ethics: beneficence, non-maleficence, justice, and autonomy [14, 15, 17, 1932, 35-39]. We therefore organized all 26 considerations not only by the context they relate to, but also by the key principle that was most applicable, as visualized in Fig. 2.

\section{Discussion}

We found 26 considerations relevant to disclosing a diagnosis of $\mathrm{AD}$ to individuals without dementia. These concerns, constraints, and implications relate to clinical, personal, and societal contexts. Many constitute direct opposites, such as certainty versus uncertainty, reflecting the heated debate among stakeholders. This duality is concordant with findings from a recent study on patients' views regarding early $A D$ diagnosis, reporting not only great variety between individuals but also profound ambivalence within individuals [41]. For example, while a diagnosis can provide certainty on what is going on, it can also bring uncertainty on what to expect. Thus, it may not have to be either one way or the other, as both sides can be true to some extent, and perspectives may change over time. This illustrates the ardent need of empirical evidence and clinical recommendations on a biomarker diagnosis of AD. Since market access has been granted to a first disease-modifying therapy [10], the urgency is even greater, as practitioners, patients, and society are presented with novel opportunities and challenges.

Particularly with respect to clinical validity, the comparison of statements was hampered by a disparity of definitions. In the absence of a gold standard, authors evaluated the accuracy of the biomarker framework [4] according to various views on the true state of AD. Findings were based on different criteria of clinical symptoms, pathological findings, and/or biological changes. While all models have value, they are not interchangeable. Moreover, studies suggest the scientific dissensus on nosology and the shifting meaning of AD create confusion [42-45]. This emphasizes the need for a common concept and language of AD [46].

An underlying and fundamentally contested conundrum is whether individuals with normal cognition, but abnormal biomarkers are ill. Based on research criteria, they have $A D$ but judging by clinical standards they are not sick [21, 27, 30-32, 38, 47]. Two of the included papers evaluate the conceptual validity according to theories of health and disease $[26,34]$. The authors reasoned that the signature of amyloid and tau does not represent

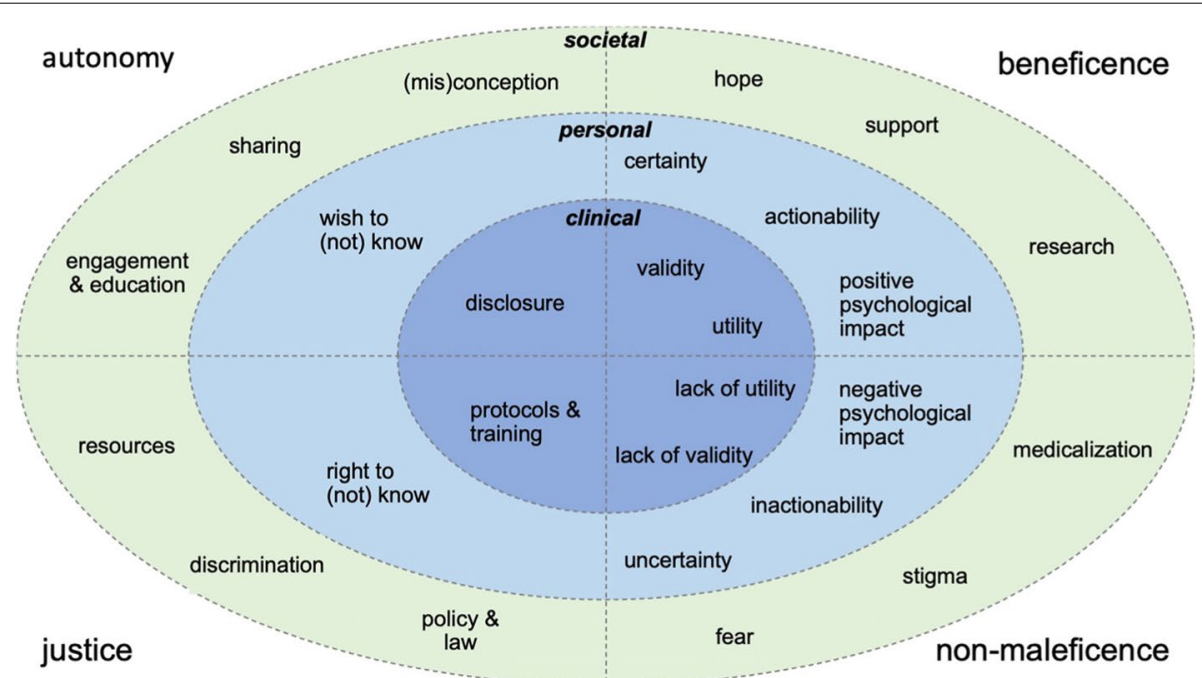

Fig. 2 Visual overview of considerations. Visual overview of 26 considerations extracted from the included literature, categorized based on the clinical, personal, or societal context they relate to and the four basic principles of biomedical ethics: beneficence (doing good), non-maleficence (avoiding harm), justice (ensuring fair distribution of resources in accordance with the law), and autonomy (allowing free, informed, and deliberate decisions). Contested issues, e.g., (in) actionability, are ranked under beneficence as well as non-maleficence to reflect the theoretical debate and their subjective nature. Societal considerations are interrelated, e.g., sharing test results can lead to support but also stigma and discrimination. The visual overview highlights the tension between clinicians' responsibility to weigh the benefits and risks and prevent unnecessary suffering, versus individuals' right to self-determination 
a singular disease, nor a statistical deviation from normal aging in older people. They concluded that people without symptoms should not be diagnosed as "patients-inwaiting," but considered persons at-risk [34]. Yet this is not about screening unsuspecting populations. Neither is the phase before dementia entirely without symptoms; individuals present at memory clinics because they experience symptoms in their daily lives, albeit subtle or mild $[48,49]$. They wish to learn what is wrong, and they have a right to know. In the field of oncology, it is common to diagnose patients with cancer (in situ), regardless of signs or complaints. The same goes for conditions like hypertension and diabetes mellitus $[50,51]$. An apparent difference is the lack of disease-modifying interventions for AD. Some ethicists apply a "pragmatic view" to AD, stating that without preventive medication early detecting may do more harm than good [34]. This view might change with the recent conditional approval of a first disease-modifying treatment by the FDA.

Overall, the identified literature tended to concentrate on putative adverse implications. Notably, repeatedly mentioned worries about conforming to stereotypes or nocebo reactions were substantiated by evidence from a single study on disclosure of genetic risk [52]. Although inherited susceptibility is beyond the scope of our review, extensive research on the impact of revealing an increased probability [53-60] or absolute certainty [6163] of developing dementia has demonstrated that catastrophic outcomes are rare, knowledge of the test results does not affect cognition, and participants also perceive benefits. So far, evidence on disclosing biomarker information is limited, but the few available studies suggest it is safe and actionable [64-69]. However, stigmatization and discrimination are concerns that need further scrutiny [70-72]. More importantly, it should be noted these findings are based on a selection of individuals, willing to participate and learn their disposition to develop dementia. There is a lack of racial, ethnical, cultural, social, economic, and environmental diversity in study populations [73]. More empirical research is required, evaluating both harms and benefits, taking perspectives of individuals from all groups into account.

The key principles of medical ethics, i.e., beneficence, non-maleficence, justice, and autonomy, were frequently invoked to decide whether a predementia diagnosis of $\mathrm{AD}$ is justified. However, applying the "four principle approach" may unduly simplify a complicated matter [74]. The framework relies on the notion of a common morality, while the interest, motivation, and implications of biomarker testing are inherently deeply personal [75]. Yet patients' perspectives and circumstances are underrepresented in the theoretical discourse. Rather than risking paternalism by imposing the moral right to know or not know on all, we need a tailored approach in clinical settings to respect the values of each individual. Future research should illuminate which personal factors influence people's preferences for medical information, as well as the psychological and social implications of disclosing test results. It is pivotal to engage and educate all stakeholders to enable informed (and shared) decision-making and empower individuals in choosing what is best for them. This becomes especially relevant in light of the development of low-cost blood tests [76, 77], advances in risk-reducing lifestyle programs [3, 78-81], and progress on disease-modifying therapies [82-85].

\section{Strengths and limitations}

Our systematic review provides an in-depth overview of considerations regarding a diagnosis of $\mathrm{AD}$ before dementia. Strengths are our broad query to include publications from various disciplines (including medical, ethical, psychological, social, and legal), strict adherence to PRISMA guidelines, and use of state-of-the art methodology to inductively analyze the literature. Among the potential limitations is the restriction to articles in English presenting theoretical data. A next step is an inventory of empirical evidence in the clinical, personal, and societal contexts to compare expectations to experiences and identify gaps in knowledge. Immediate requirements include devising educational materials for the general public, protocols for clinical practice, supportive services for patients, and legislation to protect their rights [86]. The identified considerations offer helpful starting points to prepare for a future with precision medicine and prevention of $\mathrm{AD}$.

\section{Conclusions}

Diagnosing AD in individuals without dementia involves diverse and often opposing considerations, related to a clinical, personal, or societal context. The theoretical literature tended to focus on adverse impact and rely on the notion of a common morality while the motivation for, and implications of, biomarker testing are deeply personal. Our findings provide a starting point for memory clinic specialists, such as neurologists, geriatricians, and psychiatrists, to discuss a biomarker-based diagnosis with their patients, to enable shared and informed decision-making, which will become even more relevant in light of the conditional approval of a first disease-modifying drug for AD.

\footnotetext{
Acknowledgements

Research of Alzheimer center Amsterdam is part of the neurodegeneration research program of Amsterdam Neuroscience. Alzheimer Center Amsterdam is supported by Stichting Alzheimer Nederland and Stichting VUmc fonds. WMvdF holds the Pasman Chair. JvdS is appointed at ABOARD, which is a public-private partnership receiving funding from ZonMW (\#73305095007) and Health Holland, Topsector Life Sciences \& Health (PPP-allowance;
} 
\#LSHM20106). More than 30 partners participate in ABOARD. ABOARD also receives funding from Edwin Bouw Fonds and Gieskes-Strijbisfonds. PS is a recipient of JPND-funded EURO-FINGERS (ZonMW-Memorabel \#733051102).

\section{Authors' contributions}

WMvdF and JvdS designed the study and searched the literature. JvdS and JFCK formulated the query and selected the databases. JvdS drafted the manuscript. WMvdF, ALB, and LNCV contributed to the analysis and interpretation of data. WMvdF, ALB, LNCV, JFCK, FHB, and PS critically revised the manuscript. All authors read the manuscript and approved it for submission.

\section{Funding}

JvdS is appointed at ABOARD, which is a public-private partnership receiving funding from ZonMW (\#73305095007) and Health Holland, Topsector Life Sciences \& Health (PPP-allowance; \#LSHM20106). More than 30 partners participate in ABOARD.

\section{Availability of data and materials}

The datasets used and/or analyzed during the current study are available from the corresponding author on reasonable request.

\section{Declarations}

\section{Ethics approval and consent to participate}

Not applicable.

\section{Consent for publication}

Not applicable.

\section{Competing interests}

JvdS wrote a book for a layman's audience about the personal impact of dominantly inherited Alzheimer's disease, for which she received grants or contracts from Aegon Nederland and Alzheimer Nederland and royalties from Uitgeverij Prometheus. She received compensation for writings, presentations, or educational events on this topic from Zin Magazine, Psychologie Magazine, Libelle, NRC Media, Provincie Drenthe, Radboud UMC, and Roche NL. She is a member of the advisory board for the National Dementia Strategy of the Dutch Ministry of Health, Welfare and Sport. All payments are made to her. LNCV is supported by a fellowship grant received from Alzheimer Nederland (WE.15-2019-05). She received a small fee for the development of an online course on shared decision-making by EACH, the international organization for research in healthcare. Payments were made to her institution. FHB received grants, contracts, or consulting fees from Optina Diagnostics (Canada), Biogen, and Roche. Payments were made to her institution. PS has received consultancy fees (paid to the institution) from AC Immune, Alkermes, Alnylam, Alzheon, Anavex, Axoltis, Brainstorm Cell, Cortexyme, Denali, EIP, ImmunoBrain Checkpoint, GemVax, Genentech, Green Valley, Novartis, Novo Nordisk, PeopleBio, Renew LLC, and Roche. He received payment or honoraria from Nutricia. He is PI of studies with AC Immune, CogRx, FUJI-film/Toyama, IONIS, UCB, and Vivoryon. He is a part-time employee of Life Sciences Partners Amsterdam. He serves on the board of Brain Research Center and New Amsterdam Pharma. He participated on a Data Safety Monitoring Board or Advisory Board at Genentech. He is a member of the advisory board for the National Dementia Strategy of the Dutch Ministry of Health, Welfare and Sport (paid to the institution). He is co-editor-in-chief of the Alzheimer's Research and Therapy and was firewalled from the review and decision process. ALB received grants or contracts (paid to her institution) from Horizon2020 INKplant: "Ink-based hybrid multi-material fabrication of next generation implants" (2021-2025); COGEM (RIVM Committee on Genetic Modification) Grant: "The role and meaning of the concept'naturalness' in scientific, legal and societal context" (2019-2021); Netherlands Organisation for Scientific Research (NWO) Crossover Grant:"INTENSE: Innovative Neurotechnology for Society" (2020-2025); Netherlands Organisation for Scientific Research (NWO): "RAIDIO: Responsible Artificial Intelligence for Clinical Decision-Making" (2020-2025); EU H2020 grant "EXPANSE: exposome empowered tools for healthy living in urban settings" (2020-2024); National Science Agenda (NWA): SYMPHONY: Orchestrating personalised treatment in patients with a bleeding disorder (2019-2023); Horizon2020 grant "iPSpine: induced pluripotent stem cell-based therapy for spinal regeneration" (2019-2024). She is a member of Dutch Senate (payment to her), Board of ZonMw (payment to her institution),
Supervisory Board Amsterdam UMC (payment to her) International Society for Stem Cell Research Ethics Committee (no payment), and IQVIA's Ethics Advisory Panel (payment to her institution). Research programs of WMvdF have been funded by ZonMW, NWO, EU-FP7, EU-JPND, Alzheimer Nederland, CardioVascular Onderzoek Nederland, Health Holland, Topsector Life Sciences \& Health, stichting Dioraphte, Gieskes-Strijbis fonds, stichting Equilibrio, Pasman stichting, Biogen MA Inc, Boehringer Ingelheim, Life-MI, AVID, Roche BV, Fujifilm, and Combinostics. She holds the Pasman chair. She is a consultant to Oxford Health Policy Forum CIC, Roche, and Biogen MA Inc. She participated in an advisory board of Biogen MA Inc. She is an associate editor at Brain. All funding is paid to her institution.

\section{Author details}

${ }^{1}$ Alzheimer Center Amsterdam, Department of Neurology, Amsterdam Neuroscience, Vrije Universiteit Amsterdam, Amsterdam UMC, De Boelelaan 1118, 1081, HZ, Amsterdam, The Netherlands. ${ }^{2}$ Division of Clinical Geriatrics, Center for Alzheimer Research, Department of Neurobiology, Care Sciences and Society, Karolinska Institutet, Stockholm, Sweden. ${ }^{3}$ Medical Library, Vrije Universiteit, Amsterdam, The Netherlands. ${ }^{4}$ Erasmus School of Philosophy, Erasmus University Rotterdam, Rotterdam, The Netherlands. ${ }^{5}$ Department of Epidemiology \& Data Sciences, Vrije Universiteit Amsterdam, Amsterdam UMC, Amsterdam, The Netherlands.

Received: 18 October 2021 Accepted: 31 January 2022

Published online: 10 February 2022

\section{References}

1. Bateman RJ, Xiong C, Benzinger TLS, Fagan AM, Goate A, Fox NC, et al. Clinical and biomarker changes in dominantly inherited Alzheimer's disease. N Engl J Med. 2012;367:795-804.

2. Villemagne VL, Burnham $S$, Bourgeat $P$, Brown B, Ellis KA, Salvado O, et al. Amyloid $\beta$ deposition, neurodegeneration, and cognitive decline in sporadic Alzheimer's disease: a prospective cohort study. Lancet Neurol. 2013;12:357-67.

3. Ngandu T, Lehtisalo J, Solomon A, Levälahti E, Ahtiluoto S, Antikainen $R$, et al. A 2 year multidomain intervention of diet, exercise, cognitive training, and vascular risk monitoring versus control to prevent cognitive decline in at-risk elderly people (FINGER): a randomised controlled trial. Lancet. 2015;385:2255-63.

4. Jack CR Jr, Bennett DA, Blennow K, Carrillo MC, Dunn B, Haeberlein SB, et al. NIA-AA Research Framework: toward a biological definition of Alzheimer's disease. Alzheimers Dement. 2018;14:535-62.

5. Draper B, Peisah C, Snowdon J, Brodaty H. Early dementia diagnosis and the risk of suicide and euthanasia. Alzheimers Dement. 2010:6:75-82.

6. Grill JD, Cox CG, Harkins K, Karlawish J. Reactions to learning a "not elevated" amyloid PET result in a preclinical Alzheimer's disease trial. Alzheimers Res Ther. 2018;10:125.

7. Brookmeyer R, Abdalla N, Kawas CH, Corrada MM. Forecasting the prevalence of preclinical and clinical Alzheimer's disease in the United States. Alzheimers Dement. 2018;14:121-9.

8. Gooblar J, Roe CM, Selsor NJ, Gabel MJ, Morris JC. Attitudes of research participants and the general public regarding disclosure of Alzheimer disease research results. JAMA Neurol. 2015;72:1484-90.

9. Jack CR, Therneau TM, Weigand SD, Wiste HJ, Knopman DS, Vemuri P, et al. Prevalence of biologically vs clinically defined Alzheimer spectrum entities using the National Institute on Aging-Alzheimer's Association Research Framework. JAMA Neurology. 2019;76:1174.

10. Alzforum. Aducanumab approved to treat Alzheimer's disease. 2021.

11. Moher D, Liberati A, Tetzlaff J, Altman DG. Preferred Reporting Items for Systematic Reviews and Meta-Analyses: the PRISMA statement. PLoS Med. 2009;6:e1000097.

12. Elo $\mathrm{S}$, Kyngäs $\mathrm{H}$. The qualitative content analysis process. J Adv Nurs. 2008;62:107-15.

13. Beauchamp TL, Childress JF. Principles of biomedical ethics. 8th ed; 2019.

14. Europe A. Discussion paper on ethical issues linked to the changing definitions/use of terms related to AD. Luxembourg: Alzheimer Europe; 2016. 
15. Bunnik EM, Richard E, Milne R, Schermer MHN. On the personal utility of Alzheimer's disease-related biomarker testing in the research context. J Med Ethics. 2018;44:830-4.

16. O'Brien J, Dubois B, Gauthier S, Cummings J, Chiu HF, Brodaty H. For debate: is very early diagnosis of $A D$ using the new criteria beneficial for patient. Int Psychogeriatr. 2013;25:177-81.

17. De Wilde A, Van Buchem MM, Otten RHJ, Bouwman F, Stephens A, Barkhof $F$, et al. Disclosure of amyloid positron emission tomography results to individuals without dementia: a systematic review. Alzheimers Res Ther. 2018;10(1):72.

18. Lilamand $M$, Hourregue C, Paquet C. Interest of biological biomarkers in the diagnostic approach of neurocognitive disorders in the elderly. Rev Neurol (Paris). 2020;176(9):677-83.

19. Molinuevo JL, Rami L. Applying the IWG research criteria in clinical practice: feasibility and ethical issues. Med Clin North Am. 2013;97:477-84

20. Porteri C, Albanese E, Scerri C, Carrillo MC, Snyder HM, Martensson B, et al. The biomarker-based diagnosis of Alzheimer's disease. 1-ethical and societal issues. Neurobiol Aging. 2017;52:132-40.

21. Porteri C, Frisoni GB. Biomarker-based diagnosis of mild cognitive impairment due to Alzheimer's disease: how and what to tell. A kickstart to an ethical discussion. Front Aging Neurosci. 2014;6:41.

22. Roberts JS, Dunn LB, Rabinovici GD. Amyloid imaging, risk disclosure and Alzheimer's disease: ethical and practical issues. Neurodegener Dis Manag. 2013;3:219-29.

23. Smedinga M, Tromp K, Schermer MHN, Richard E. Ethical arguments concerning the use of Alzheimer's disease biomarkers in individuals with no or mild cognitive impairment: a systematic review and framework for discussion. J Alzheimers Dis. 2018;66:1309-22.

24. Vanderschaeghe $G$, Dierickx K, Vandenberghe R. Review of the ethical issues of a biomarker-based diagnoses in the early stage of Alzheimer's disease. J Bioeth Inq. 2018;15:219-30.

25. Alpinar-Sencan Z, Schicktanz S. Addressing ethical challenges of disclosure in dementia prediction: limitations of current guidelines and suggestions to proceed. BMC Medical Ethics. 2020;21:33.

26. Alexopoulos P, Kurz A. The new conceptualization of Alzheimer's disease under the microscope of influential definitions of disease. Psychopathology. 2015;48:359-67.

27. Chetelat G, La Joie R, Villain N, Perrotin A, de La Sayette V, Eustache F, et al. Amyloid imaging in cognitively normal individuals, at-risk populations and preclinical Alzheimer's disease. Neuroimage Clin. 2013;2:356-65.

28. Ahlgrim NS, Garza K, Hoffman C, Rommelfanger KS. Prodromes and preclinical detection of brain diseases: surveying the ethical landscape of predicting brain health. eneuro. 2019;6(4):ENEURO.0439-18.2019.

29. Dubois B, Hampel H, Feldman HH, Scheltens P, Aisen P, Andrieu S, et al. Preclinical Alzheimer's disease: definition, natural history, and diagnostic criteria. Alzheimers Dement. 2016;12:292-323.

30. Gauthier S, Leuzy A, Racine E, Rosa-Neto P. Diagnosis and management of Alzheimer's disease: past, present and future ethical issues. Prog Neurobiol. 2013:110:102-13.

31. Grill JD, Johnson DK, Burns JM. Should we disclose amyloid imaging results to cognitively normal individuals? Neurodegenerative Dis Manag. 2013;3:43-51.

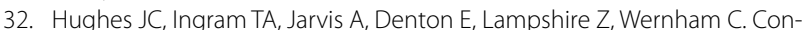
sent for the diagnosis of preclinical dementia states: a review. Maturitas. 2017:98:30-4.

33. Lawrence MW, Arias JJ. Alzheimer's disease biomarkers: another tool for FAA pilot screening? J Law Biosci. 2019;6:85-110.

34. Schermer MHN, Richard E. On the reconceptualization of Alzheimer's disease. Bioethics. 2019;33:138-45.

35. Schicktanz S, Schweda M, Ballenger JF, Fox PJ, Halpern J, Kramer JH, et al. Before it is too late: professional responsibilities in late-onset Alzheimer's research and pre-symptomatic prediction. Front Hum Neurosci. 2014;8:921

36. Erdmann $P$, Langanke $M$. The ambivalence of early diagnosis - returning results in current Alzheimer research. Curr Alzheimer Res. 2018;15:28-37.

37. Stites SD, Milne R, Karlawish J. Advances in Alzheimer's imaging are changing the experience of Alzheimer's disease. Alzheimers Dement (Amst). 2018;10:285-300.

38. Johnson RA, Karlawish J. A review of ethical issues in dementia. Int Psychogeriatr. 2015;27:1635-47.
39. Karlawish J. Addressing the ethical, policy, and social challenges of preclinical Alzheimer disease. Neurology. 2011;77:1487-93.

40. Leuzy A, Gauthier S. Ethical issues in Alzheimer's disease: an overview. Expert Rev Neurother. 2012;12:557-67.

41. Nielsen KD, Boenink M. Ambivalent anticipation: how people with Alzheimer's disease value diagnosis in current and envisioned future practices. Sociol Health IIIn. 2021;43:510-27.

42. Beard RL, Neary TM. Making sense of nonsense: experiences of mild cognitive impairment. Sociol Health IIIn. 2013;35:130-46.

43. Lingler JH, Nightingale MC, Erlen JA, Kane AL, Reynolds CF, Schulz R, et al. Making sense of mild cognitive impairment: a qualitative exploration of the patient's experience. Gerontologist. 2006;46:791-800.

44. Gomersall T, Astell A, Nygård L, Sixsmith A, Mihailidis A, Hwang A. Living with ambiguity: a metasynthesis of qualitative research on mild cognitive impairment. Gerontologist. 2015;55:892-912.

45. Gomersall T, Smith SK, Blewett C, Astell A. 'It's definitely not Alzheimer's': perceived benefits and drawbacks of a mild cognitive impairment diagnosis. Br J Health Psychol. 2017;22:786-804.

46. Knopman DS, Petersen RC, Jack CR. A brief history of "Alzheimer disease". Neurology. 2019;92(22):1053-59.

47. Dubois B, Villain N, Frisoni GB, Rabinovici GD, Sabbagh M, Cappa S, et al. Clinical diagnosis of Alzheimer's disease: recommendations of the International Working Group. Lancet Neurol. 2021;20(6):484-96.

48. Jessen F, Amariglio RE, Buckley RF, van der Flier WM, Han Y, Molinuevo JL, et al. The characterisation of subjective cognitive decline. Lancet Neurol. 2020;19:271-8.

49. Cecchini MA, Yassuda MS, Squarzoni P, Coutinho AM, de Paula FD, Duran FLS, et al. Deficits in short-term memory binding are detectable in individuals with brain amyloid deposition in the absence of overt neurodegeneration in the Alzheimer's disease continuum. Brain Cogn. 2021;152:105749.

50. Gauer R. Severe asymptomatic hypertension: evaluation and treatment. Am Fam Physician. 2017:95:492-500.

51. American Diabetes Association. 2. Classification and diagnosis of diabetes: standards of medical care in diabetes - 2020. Diabetes Care. 2020:43:S14.

52. Lineweaver TT, Bondi MW, Galasko D, Salmon DP. Effect of knowledge of APOE genotype on subjective and objective memory performance in healthy older adults. Am J Psychiatr. 2014;171:201-8.

53. Chao S, Roberts JS, Marteau TM, Silliman R, Cupples LA, Green RC. Health behavior changes after genetic risk assessment for Alzheimer disease: the REVEAL study. Alzheimer Dis Assoc Disord. 2008:22:94-7.

54. Christensen KD, Roberts JS, Uhlmann WR, Green RC. Changes to perceptions of the pros and cons of genetic susceptibility testing after APOE genotyping for Alzheimer disease risk. Genet Med. 2011;13:409-14.

55. Eckert SL, Katzen H, Roberts JS, Barber M, Ravdin LD, Relkin NR, et al. Recall of disclosed Apolipoprotein E genotype and lifetime risk estimate for Alzheimer's disease: The REVEAL Study. Genet Med. 2006;8:746-51.

56. Green RC, Roberts JS, Cupples LA, Relkin NR, Whitehouse PJ, Brown T, et al. Disclosure of APOE genotype for risk of Alzheimer's disease. N Engl J Med. 2009:361:245-54.

57. Linnenbringer E, Roberts JS, Hiraki S, Cupples LA, Green RC."I know what you told me, but this is what I think:" perceived risk of Alzheimer disease among individuals who accurately recall their genetics-based risk estimate. Genet Med. 2010;12:219-27.

58. Roberts J, Christensen K, Green R. Using Alzheimer's disease as a model for genetic risk disclosure: implications for personal genomics. Clin Genet. 2011:80:407-14.

59. Roberts JS, Cupples LA, Relkin NR, Whitehouse PJ, Green RC. Genetic risk assessment for adult children of people with Alzheimer's disease: the Risk Evaluation and Education for Alzheimer's Disease (REVEAL) study. J Geriatr Psychiatry Neurol. 2005;18:250-5.

60. Zick CD, Mathews CJ, Roberts JS, Cook-Deegan R, Pokorski RJ, Green RC. Genetic testing for Alzheimer's disease and its impact on insurance purchasing behavior. Health Aff. 2005;24:483-90.

61. Cohn-Hokke PE, van Swieten JC, Pijnenburg YAL, Tibben A, MeijersHeijboer $\mathrm{H}$, Kievit A. The effect of predictive testing in adult-onset neurodegenerative diseases on social and personal life. J Genet Couns. 2018:27:947-54.

62. Paulsen JS, Nance M, Kim J-I, Carlozzi NE, Panegyres PK, Erwin $\mathrm{C}$, et al. A review of quality of life after predictive testing for and 
earlier identification of neurodegenerative diseases. Prog Neurobiol. 2013;110:2-28.

63. Steinbart EJ, Smith CO, Poorkaj P, Bird TD. Impact of DNA testing for earlyonset familial Alzheimer disease and frontotemporal dementia. Arch Neurol. 2001;58:1828-31.

64. Burns JM, Johnson DK, Liebmann EP, Bothwell RJ, Morris JK, Vidoni ED. Safety of disclosing amyloid status in cognitively normal older adults. Alzheimers Dement. 2017;13:1024-30.

65. Grill JD, Raman R, Ernstrom K, Sultzer DL, Burns JM, Donohue MC, et al. Short-term psychological outcomes of disclosing amyloid imaging results to research participants who do not have cognitive impairment. JAMA Neurol. 2020.

66. Lim YY, Maruff P, Getter C, Snyder PJ. Disclosure of positron emission tomography amyloid imaging results: a preliminary study of safety and tolerability. Alzheimers Dement. 2016;12:454-8.

67. Wake T, Tabuchi H, Funaki K, Ito D, Yamagata B, Yoshizaki T, et al. The psychological impact of disclosing amyloid status to Japanese elderly: a preliminary study on asymptomatic patients with subjective cognitive decline. Int Psychogeriatr. 2018;30:635-9.

68. Aschenbrenner AJ, James BD, McDade E, Wang G, Lim YY, Benzinger TLS, et al. Awareness of genetic risk in the Dominantly Inherited Alzheimer Network (DIAN). Alzheimers Dementia. 2020;16:219-28.

69. Erickson CM, Chin NA, Johnson SC, Gleason CE, Clark LR. Disclosure of preclinical Alzheimer's disease biomarker results in research and clinical settings: why, how, and what we still need to know. Alzheimers Dementia. 2021;13(1):e12150.

70. Tibben A, Stevens M, de Wert GM, Niermeijer MF, van Duijn CM, van Swieten JC. Preparing for presymptomatic DNA testing for early onset Alzheimer's disease/cerebral haemorrhage and hereditary Pick disease. J Med Genet. 1997;34:63-72.

71. Largent EA, Harkins K, van Dyck CH, Hachey S, Sankar P, Karlawish J. Cognitively unimpaired adults' reactions to disclosure of amyloid PET scan results. PLoS One. 2020;15:e0229137.

72. Milne R, Bunnik E, Diaz A, Richard E, Badger S, Gove D, et al. Perspectives on communicating biomarker-based assessments of Alzheimer's disease to cognitively healthy individuals2. J Alzheimers Dis. 2018;62:487-98.

73. Tsoy E, Kiekhofer RE, Guterman EL, Tee BL, Windon CC, Dorsman KA, et al. Assessment of racial/ethnic disparities in timeliness and comprehensiveness of dementia diagnosis in California. JAMA Neurol. 2021;78:657-65.

74. Bredenoord A. The principles of biomedical ethics revisited; 2016. p. 133-51.

75. Vanderschaeghe $G$, Vandenberghe R, Dierickx K. Stakeholders' views on early diagnosis for Alzheimer's disease, clinical trial participation and amyloid PET disclosure: a focus group study. J Bioeth Inq. 2019;16:45-59.

76. Nakamura A, Kaneko N, Villemagne VL, Kato T, Doecke J, Doré V, et al. High performance plasma amyloid- $\beta$ biomarkers for Alzheimer's disease. Nature. 2018:554:249-54.

77. Thijssen EH, La Joie R, Wolf A, Strom A, Wang P, laccarino L, et al. Diagnostic value of plasma phosphorylated tau181 in Alzheimer's disease and frontotemporal lobar degeneration. Nat Med. 2020;26:387-97.

78. Solomon A, Turunen H, Ngandu T, Peltonen M, Levälahti E, Helisalmi S, et al. Effect of the apolipoprotein E genotype on cognitive change during a multidomain lifestyle intervention. JAMA Neurology. 2018;75:462.

79. Andrieu S, Guyonnet S, Coley N, Cantet C, Bonnefoy M, Bordes S, et al. Effect of long-term omega 3 polyunsaturated fatty acid supplementation with or without multidomain intervention on cognitive function in elderly adults with memory complaints (MAPT): a randomised, placebocontrolled trial. Lancet Neurol. 2017;16:377-89.

80. Delrieu J, Payoux P, Carrié I, Cantet C, Weiner M, Vellas B, et al. Multidomain intervention and/or omega-3 in nondemented elderly subjects according to amyloid status. Alzheimers Dementia. 2019;15:1392-401.

81. Van Charante EPM, Richard E, Eurelings LS, Van Dalen J-W, Ligthart SA, Van Bussel EF, et al. Effectiveness of a 6 -year multidomain vascular care intervention to prevent dementia (preDIVA): a cluster-randomised controlled trial. Lancet. 2016;388:797-805.

82. Sevigny J, Chiao P, Bussière T, Weinreb PH, Williams L, Maier M, et al. The antibody aducanumab reduces $A \beta$ plaques in Alzheimer's disease. Nature. 2016;537:50-6.

83. Mintun MA, Lo AC, Duggan Evans C, Wessels AM, Ardayfio PA, Andersen SW, et al. Donanemab in early Alzheimer's disease. N Engl J Med. 2021:384(18):1691-704.
84. Tolar M, Abushakra S, Hey JA, Porsteinsson A, Sabbagh M. Aducanumab, gantenerumab, BAN2401, and ALZ-801 - the first wave of amyloidtargeting drugs for Alzheimer's disease with potential for near term approval. Alzheimers Res Ther. 2020;12.

85. Swanson CJ, Zhang Y, Dhadda S, Wang J, Kaplow J, Lai RYK, et al. A randomized, double-blind, phase $2 \mathrm{~b}$ proof-of-concept clinical trial in early Alzheimer's disease with lecanemab, an anti-A $\beta$ protofibril antibody. Alzheimers Res Ther. 2021;13(1):80.

86. Arias JJ, Karlawish J. Confidentiality in preclinical Alzheimer disease studies: when research and medical records meet. Neurology. 2014;82:725-9.

\section{Publisher's Note}

Springer Nature remains neutral with regard to jurisdictional claims in published maps and institutional affiliations.
Ready to submit your research? Choose BMC and benefit from:

- fast, convenient online submission

- thorough peer review by experienced researchers in your field

- rapid publication on acceptance

- support for research data, including large and complex data types

- gold Open Access which fosters wider collaboration and increased citations

- maximum visibility for your research: over $100 \mathrm{M}$ website views per year

At BMC, research is always in progress.

Learn more biomedcentral.com/submissions 